\title{
BMJ Open STEMS pilot trial: a pilot cluster randomised controlled trial to investigate the addition of patient direct access to physiotherapy to usual GP-led primary care for adults with musculoskeletal pain
}

\author{
Annette Bishop, ${ }^{1}$ Reuben O Ogollah, ${ }^{1}$ Sue Jowett, ${ }^{2}$ Jesse Kigozi, ${ }^{2}$ \\ Stephanie Tooth, ${ }^{1}$ Joanne Protheroe, ${ }^{1}$ Elaine M Hay, ${ }^{1}$ Chris Salisbury, ${ }^{3}$ \\ Nadine E Foster, ${ }^{1}$ and the STEMS study team
}

To cite: Bishop A, Ogollah R0, Jowett S, et al. STEMS pilot trial: a pilot cluster randomised controlled trial to investigate the addition of patient direct access to physiotherapy to usual GP-led primary care for adults with musculoskeletal pain. BMJ Open 2017;7: e012987. doi:10.1136/ bmjopen-2016-012987

- Prepublication history and additional material is available. To view please visit the journal (http://dx.doi.org/ 10.1136/bmjopen-2016012987).

Received 12 July 2016 Revised 4 January 2017 Accepted 7 February 2017

CrossMark

For numbered affiliations see end of article.

Correspondence to Dr Annette Bishop; a.bishop@keele.ac.uk

\section{ABSTRACT}

Introduction: Around $17 \%$ of general practitioner (GP) consultations are for musculoskeletal conditions, which will rise as the population ages. Patient direct access to physiotherapy provides one solution, yet adoption in the National Health Service (NHS) has been slow.

Setting: A pilot, pragmatic, non-inferiority, cluster randomised controlled trial (RCT) in general practice and physiotherapy services in the UK.

Objectives: Investigate feasibility of a main RCT. Participants: Adult patients registered in participating practices and consulting with a musculoskeletal problem.

Interventions: 4 general practices (clusters) randomised to provide GP-led care as usual or the addition of a patient direct access to physiotherapy pathway.

Outcomes: Process outcomes and exploratory analyses of clinical and cost outcomes.

Data collection: Participant-level data were collected via questionnaires at identification, 2, 6 and 12 months and through medical records.

Blinding: The study statistician and research nurses were blinded to practice allocation.

Results: Of 2696 patients invited to complete study questionnaires, 978 participated (intervention group $n=425$, control arm $n=553$ ) and were analysed. Participant recruitment was completed in 6 months. Follow-up rates were $78 \%$ (6 months) and $71 \%$ (12 months). No evidence of selection bias was observed. The direct access pathway was used by $90 \%$ of patients in intervention practices needing physiotherapy. Some increase in referrals to physiotherapy occurred from one practice, although waiting times for physiotherapy did not increase (28 days before, 26 days after introduction of direct access). No safety issues were identified. Clinical and cost outcomes were similar in both groups.

\section{Strengths and limitations of this study}

- This was a pilot randomised controlled trial (RCT) of patient direct access to physiotherapy in four general practices and one physiotherapy service in one English town.

- It provides evidence for the feasibility and safety of patient direct access to physiotherapy services.

- Observed recruitment and follow-up rates can inform a future full RCT.

- As a pilot RCT, analyses of clinical and cost outcomes are exploratory.

Exploratory estimates of between group effect (using 36-item Short Form Health Survey (SF-36) Physical Component Summary (PCS)) at 6 months was -0.28 $(95 \% \mathrm{Cl}-1.35$ to 0.79$)$ and at 12 months $0.12(95 \%$ $\mathrm{Cl}-1.27$ to 1.51 ).

Conclusions: A full RCT is feasible and will provide trial evidence about the clinical and cost-effectiveness of patient direct access to physiotherapy.

Trial registration number: ISRCTN23378642.

\section{BACKGROUND}

In the UK, primary care is struggling to meet the current record demand from patients. General practitioners (GPs) now do an estimated 370 million consultations each year, 60 million more than 5 years ago, ${ }^{1}$ which equates to a $16 \%$ increase in workload since $2007 .^{2}$ Musculoskeletal problems account for around $17 \%$ of GP consultations ${ }^{3}$ and given the ageing population, the demand for musculoskeletal healthcare is set to rise further. ${ }^{4}$ 
Patient direct access to UK National Health Service (NHS) physiotherapy is one potential solution, allowing patients to refer themselves to physiotherapy services rather than referral being initiated by the GP. Despite reported benefits from observational research such as reduction in GP workload particularly for repeat consultations, ${ }^{5}$ improved access for patients, ${ }^{6} 7$ and reductions in costs, ${ }^{8} 9$ progress towards widespread provision of patient direct access to NHS physiotherapy has been slow.

In the UK healthcare under the NHS is free at point of delivery, meaning that patients do not pay fees to see NHS GPs, NHS physiotherapists or to access specialist NHS hospital services. However, patients can access private healthcare providers but these costs must be met in full by the patient or by employer provided or personally bought private medical insurance. Around $11 \%$ of the UK population has some private medical insurance but many of these policies exclude many health conditions. ${ }^{10}$ The NHS is therefore the predominant healthcare provider in the UK. Legislation has allowed patients to access physiotherapists directly since 1977 but in the NHS services are commissioned by clinical commissioning groups, allocated funds from the Department of Health, and patient direct access to physiotherapy is only available in some areas of the UK. The physiotherapists involved in this pilot trial did not have advanced practice roles and so were not able to order and interpret investigations or make referrals to hospital specialist services.

A specific recommendation in the recent Primary Care Workforce Commission is to investigate whether direct access to physiotherapy for some conditions makes cost-effective use of NHS resources. ${ }^{11}$

The aim of the STEMS pilot trial was to assess the feasibility of a future large trial to compare the clinical and cost-effectiveness of the addition of patient direct access to physiotherapy versus continuing with usual GP-led care alone for adults with common musculoskeletal problems. The specific objectives of this pilot trial comprised process objectives to assess the feasibility of a future large cluster randomised trial, and research objectives that focused on exploratory analysis of clinical outcomes and costs.

\section{Process objectives}

i. To assess the engagement of GP practices and physiotherapy services to participate and stay in the trial through follow-up.

ii. To assess the feasibility of establishing a patient's direct access to physiotherapy pathway.

iii. To develop and test approaches to marketing the direct access pathway to ensure a sufficient proportion of patients use the pathway.

iv. To estimate recruitment rates in the control and intervention practices.

v. To explore any evidence of selection bias in the control and intervention practices.

vi. To estimate follow-up rates at each follow-up time point.
Research objectives

i. To explore changes in the proposed main trial primary outcome measure (physical health measured using the 36-item Short Form Health Survey (SF-36)v2 Physical Component Summary (PCS)).

ii. To explore changes in other outcome measures (overall perceived change, SF-36v2 Mental Component Summary, pain self-efficacy, quality of life, understanding of the condition, experience of care, and convenience and accessibility of services).

iii. To provide an exploratory estimate of costs, both healthcare and societal costs.

iv. To estimate the parameters needed for a realistic sample size calculation for a future large cluster RCT.

v. To explore the use of willingness to pay methodology to capture the strength of patient preferences for self-referral to NHS physiotherapy. This analysis will be reported in a separate future paper.

\section{METHODS}

\section{Design and setting}

STEMS was a pilot, pragmatic, two-arm parallel, noninferiority, cluster RCT in four general practices in one town in Cheshire, UK, referring patients to a local NHS physiotherapy service, which did not offer the patient direct access to care prior to the set-up of the STEMS trial. The methods have been described in full in the STEMS trial protocol, ${ }^{12}$ but are summarised below.

\section{Randomisation}

General practices were randomised using computergenerated random numbers stratified by the practice size (small or large, based on the average number of patients registered per practice at the start of the pilot trial) in the ratio 1:1 to continue to offer GP-led care alone as usual care $(n=2)$ or GP-led care plus the addition of a new pathway permitting patient direct access to an existing NHS physiotherapy service. Patients followed the care to which their practice was randomised and were recruited through identical patient information explaining that musculoskeletal services were being evaluated using patient self-reported clinical outcomes and medical record review. Patient direct access to physiotherapy was not available to patients registered with control practices. The study statistician was blinded to practice allocation.

\section{Participants}

Data were collected from individual participants who were eligible if they were 18 years or over and presenting to their general practice or physiotherapy service with a musculoskeletal condition. Patients who were undergoing palliative care, had severe learning disabilities, were housebound or in nursing home accommodation or unable to communicate in English were not eligible to take part. 
Potentially eligible participants were identified when they presented to the general practice or physiotherapy service and were mailed a study pack including the first questionnaire. Follow-up data collection from consenting participants was via participant self-completion postal questionnaires at 2, 6 and 12 months and through review of general practice and physiotherapy records.

\section{Setting up the patient direct access to physiotherapy pathway}

Physiotherapists attended a 1-day training programme to prepare them for providing care to patients who self-refer, including topics such as prevalence and identification of red flags, physical and musculoskeletal manifestations of underlying medical pathology and review of common over-the-counter and prescribed medications for musculoskeletal pain. ${ }^{12}$ Physiotherapists also attended regular mentoring meetings with a senior physiotherapist in the participating physiotherapy service.

The patient's direct access pathway began 8 weeks prior to the start of participant recruitment to the study in order to allow familiarisation with receiving and managing patients using the direct access pathway. All referrals (GPs or patient self-referral) were triaged using existing physiotherapy service criteria and were classified as urgent, routine or inappropriate for physiotherapy. Whether self-referring or GP referred to physiotherapy patients received equitable treatment such as the length of wait to first appointment once logged on the physiotherapy administration system. As this trial investigated the addition of direct access to physiotherapy, the physiotherapy care provided was determined by clinical need and assessment findings and was therefore consistent with routine practice and did not differ for patients in the different arms of the trial.

In order to ensure that patients were aware of the new direct access pathway, all adult registered patients at the two intervention practices $(n=8222)$ were individually mailed an information letter about direct access. Posters, flyers and self-referral forms were readily available in intervention practices. Even with the offer of self-referral, we anticipated that some patients would continue to consult their GP or practice nurse, therefore in the intervention practices, patients could access physiotherapy either by traditional written referral, be recommended to self-refer (GP or nurse 'recommended' self-referral) or choose to self-refer to physiotherapy without contacting their GP practice ('true' self-referrers).

Safety of the pathway was explored by the number of adverse events reported and by systematically searching for diagnostic codes of a wide range of pathologies (including cancer, fractures, joint infections and stroke) in the medical records of participants who used the direct access pathway.

\section{Outcomes and analysis}

As a pilot trial, the emphasis was on process outcomes to establish the feasibility for a full trial. Outcomes were collected from several sources. Anonymised process outcome data were collected from physiotherapy service records and self-referral forms. Individual participants provided data on their characteristics, clinical outcomes and costs through self-completed questionnaires following recruitment to the research and at 2, 6 and 12 months. Key outcome data were sought from nonresponders through minimum data collection (MDC). A list of outcomes is provided in table 1 . To ensure transparency in analyses, a statistical analysis plan was developed and approved by the Trial Steering Committee (TSC) prior to the final analysis. Since this was a pilot trial, the aim of all analyses was to decide if a main trial is feasible and desirable, in addition to finalising the sample size for a future main trial. Descriptive summaries report the number of participants identified and recruited, along with the number of participants followed up at each time point. Withdrawals (and where possible, reasons for withdrawals) are reported. Anonymised data on key baseline characteristics (age, gender and Index of Multiple Deprivation) of those who were invited but did not participate are compared with those who did participate.

The feasibility of offering direct access was assessed, including the number of participants who were referred to physiotherapy by their GP, those who were 'recommended self-referrals' (prompted by their GP or practice nurse to refer) and those who were 'true self-referrals' and the waiting time to the first physiotherapy appointment.

Analyses were conducted for the clinical outcomes, but this was treated as exploratory and mainly descriptive. A baseline table compared the demographic and clinical characteristics of participants. Since this is a pilot trial with only four clusters randomised, some imbalance between participants in each of the treatment arms on one or more baseline characteristics was anticipated. The baseline table was scrutinised for any serious imbalances in observable baseline variables and the trends of the imbalance if any. Recruitment rates were also estimated and compared between the control and intervention arms.

All continuous variables were summarised using mean, $\mathrm{SD}$, median and IQR as appropriate and the frequency and percentages of observed levels reported for all categorical measures. As this was a pilot trial, no emphasis was put on the $p$ values for inferential statistical tests conducted. A mixed-effect model, which allowed all available data at all the four time points to be used and accounted for missing data and clustering effect, was used to estimate a two-sided $95 \%$ CI to show a credible range for the true difference in the SF-36v2 PCS subscale between intervention and the control arms. The model was adjusted for key patient-level baseline characteristics (age, area-level deprivation and widespread pain (from manikin data)) and a random effect for the GP practices and included a treatment-by-time interaction to obtain the estimates of treatment effect (and 95\% CI) at each follow-up time point (2, 6 and 
Table 1 STEMS pilot trial questionnaire measures

Baseline 2 months 6 months 12 months

Likely primary outcome measure for main trial

Physical function

SF-36v2 Physical Component Summary ${ }^{13}$ http:// $\checkmark$ www.sf-36.org/

(the higher the score the better the physical

health)

Secondary outcome measures

Overall change in condition Global Assessment of Change since baseline- $X$ single question

Mental health

SF-36v2 Mental Component Summary ${ }^{13}$ http:// $\quad \checkmark$ www.sf-36.org/

(the higher the score the better the mental health)

Quality of life

EuroQol EQ-5D-5L http://www.euroqol.org ${ }^{14}$

(the higher the score the better the quality of life)

Self-efficacy

Pain Self-Efficacy Questionnaire ${ }^{15}$

(10-item scale: $0=$ not at all confident,

$60=$ completely confident despite pain)

Understanding of condition General Practice Assessment Questionnaire enablement subscale ${ }^{16}$

(very well, unsure, not very well)

Experience of consultations General Practice Assessment Questionnaire communication $^{16}$

(Based on 5 items represented as a percentage of the maximum possible score 100)

Convenience of services

Single question

(0-10 Numerical Rating Scale: $0=$ not at all convenient, $10=$ very convenient)

Satisfaction with services

Single question

(0-10 Numerical Rating Scale: $0=$ not satisfied, $10=$ very satisfied)

\section{Baseline measures Demographics} Baseline risk of persistent
problems

Pain location

Pain duration

Comorbidities

Economic outcomes

Further healthcare usage

Work absence

Presenteeism
Gender, date of birth, ethnicity, education, health literacy, employment status, socioeconomic status (recent paid job title, housing)

STarT Musc tool (draft tool developed at Keele University to identify patients' risk of persistent pain and disability)

Body manikin

Single question about duration of pain

Single question

Consultations, investigations, procedures, admissions, over-the-counter medications Single question (number of days) Single work performance question (0-10 Numerical Rating Scale: $0=$ not at all affected, $10=$ unable to do my job)
12 months). Analyses of the secondary outcomes were performed similarly.

The pilot data provided information on the parameters needed for a realistic sample size calculation (follow-up rates, average cluster size) for a future, main cluster RCT. Previous research in musculoskeletal disorders has estimated a minimal clinically important difference from 2 to 4 points for the SF-36v.2 PCS subscale which should be used to inform the non-inferiority margin of any future main trial. ${ }^{17-20}$ Previous research and our own previous trials have also estimated the intracluster correlation coefficient (ICG) for the PCS to inform a future main trial.

The economic analysis was exploratory, with the aim to inform the design of a full cost-utility analysis alongside a future main trial. All patients were asked to complete the five-level version of the EuroQoL-5D (EQ-5D) questionnaire at baseline, 6 months and 12 months in 
order for the quality-adjusted life years (QALYs) over the 12-month time period to be calculated for each participant. The QALYs combine information on health-related quality of life and survival. A costconsequence analysis is reported, describing all the important results relating to costs and consequences. Analyses were mainly descriptive and total costs and outcomes were summarised using means and $95 \%$ CIs. The base case cost analysis adopted a NHS and personal social services (PSS) perspective. A broader costing perspective was considered in a sensitivity analysis, taking into account NHS/PSS costs and patients' personal expenditure costs. Costs associated with work loss were also reported as part of the cost-consequence analysis.

\section{Sample size}

As this was a pilot trial, formal sample size calculation was not carried out. The sample size was based on the number of patients we anticipated could be recruited within 6 months and that was sufficiently large to estimate the key feasibility parameters (eg, recruitment and retention) to a sufficient degree of accuracy $(<10 \%$ in the rates). We aimed to recruit 960 participants at baseline, over a period of 6 months and achieve at least $80 \%$ follow-up rate $(n=768)$ at 6 months.

\section{Trial registration}

The STEMS pilot trial is registered at Current Controlled Trials ISRCTN23378642.

\section{RESULTS}

A flow chart showing the flow of practices (units of randomisation) and flow of individual participants is shown in figure 1. As can be seen one of the control practices was considerably larger than the other randomised practices resulting in more participants in the control arm in this pilot trial.

\section{Process results}

Engagement of GP practices and the physiotherapy service

Of the five GP practices approached, four participated with three full-time equivalent GPs at each of the two intervention practices and five and two in the control practices. In the physiotherapy service, of the physiotherapists who undertook the STEMS training programme, four worked in the clinic local to the participating general practices and delivered care to patients using the direct access pathway (NHS Agenda for Change band 7 $(\mathrm{n}=1)$, band $6(\mathrm{n}=2)$ and band $5(\mathrm{n}=1))$. Agenda for Change is the NHS Terms and Conditions of Service and determines salary scales for NHS staff.

\section{Feasibility of the patient direct access pathway}

Of the participants in the STEMS pilot trial $(\mathrm{n}=978)$, a total of $256(26.2 \%)$ accessed physiotherapy care during the study period, $152(35.8 \%)$ in the intervention arm and $104(18.8 \%)$ in the control arm. Of the 152 who accessed physiotherapy care in the intervention arm, $142(93.4 \%)$ were self-referrals (comprising $98(69 \%)$ 'recommended' and 44 (31\%) 'true' self-referrals).

Comparisons to service-level data (which may not be entirely comparable, due to the introduction of new administration software in the physiotherapy service), from the same period 1 year prior to start of direct access suggests one practice in the intervention arm had broadly similar rates of patients accessing physiotherapy care, whereas the other had a substantial increase once the direct access pathway had been introduced. The referral rates to physiotherapy prior to the trial showed considerable variation between the four participating practices. The practice in which referrals increased following the introduction of patient direct access previously referred 32.6 referrals per annum per 1000 registered adults compared with the other practices (59.3, 69.5 and 92.4 referrals per annum per 1000 registered adults). However, the median (IQR; range) overall waiting time in the physiotherapy service (applying to all patients in the service, as all patients joined one waiting list), from logging of the referral to first appointment, was $28(13-36 ; 0-73)$ days prior to the introduction of direct access and 26 (17-42; 0-182) days during the direct access period of the trial. Of the patients using the direct access pathway, triaging according to preexisting service criteria classified 23\% ( $\mathrm{n}=159)$ cases as urgent, $73 \%(\mathrm{n}=507)$ as routine and $4 \%(\mathrm{n}=25)$ as unsuitable for physiotherapy.

Marketing of the direct access pathway was successful. We aimed to ensure a minimum of $20 \%$ of the physiotherapy caseload during the pilot trial, from intervention arm practices, was through direct access. Using anonymised physiotherapy service data, of 765 patients registered at intervention practices and accessing care for 10 months following the introduction of the direct access pathway, $691(90.3 \%)$ used the pathway (comprising $515(74.5 \%)$ 'recommended' and $176(25.5 \%)$ 'true' self-referrals).

\section{Safety of direct access}

No adverse events were reported by GPs or physiotherapists during the pilot trial. Review of medical records identified no evidence of missed serious pathology in patients who directly accessed physiotherapy.

\section{Participant recruitment and retention}

Of 2696 participants invited to take part in the data collection, $978(36 \%)$ participated $(425(44 \%)$ in the intervention practices and $553(57 \%)$ in the control practices). Recruitment was completed within 6 months (June to December 2013). Response rates were as follows: $74.3 \%$ at 2 months (intervention $70.4 \%$, control $77.4 \%$ ), $78.4 \%$ at 6 months (intervention $76.7 \%$, control $79.8 \%$ ) and $70.7 \%$ at 12 months (intervention $69.4 \%$, control $71.6 \%$ ). MDC was included at 6 and 12 months only resulting in higher response rates at these time points. 
Figure 1 STEMS pilot trial flow chart. GP, general practitioner; MSK, musculoskeletal.

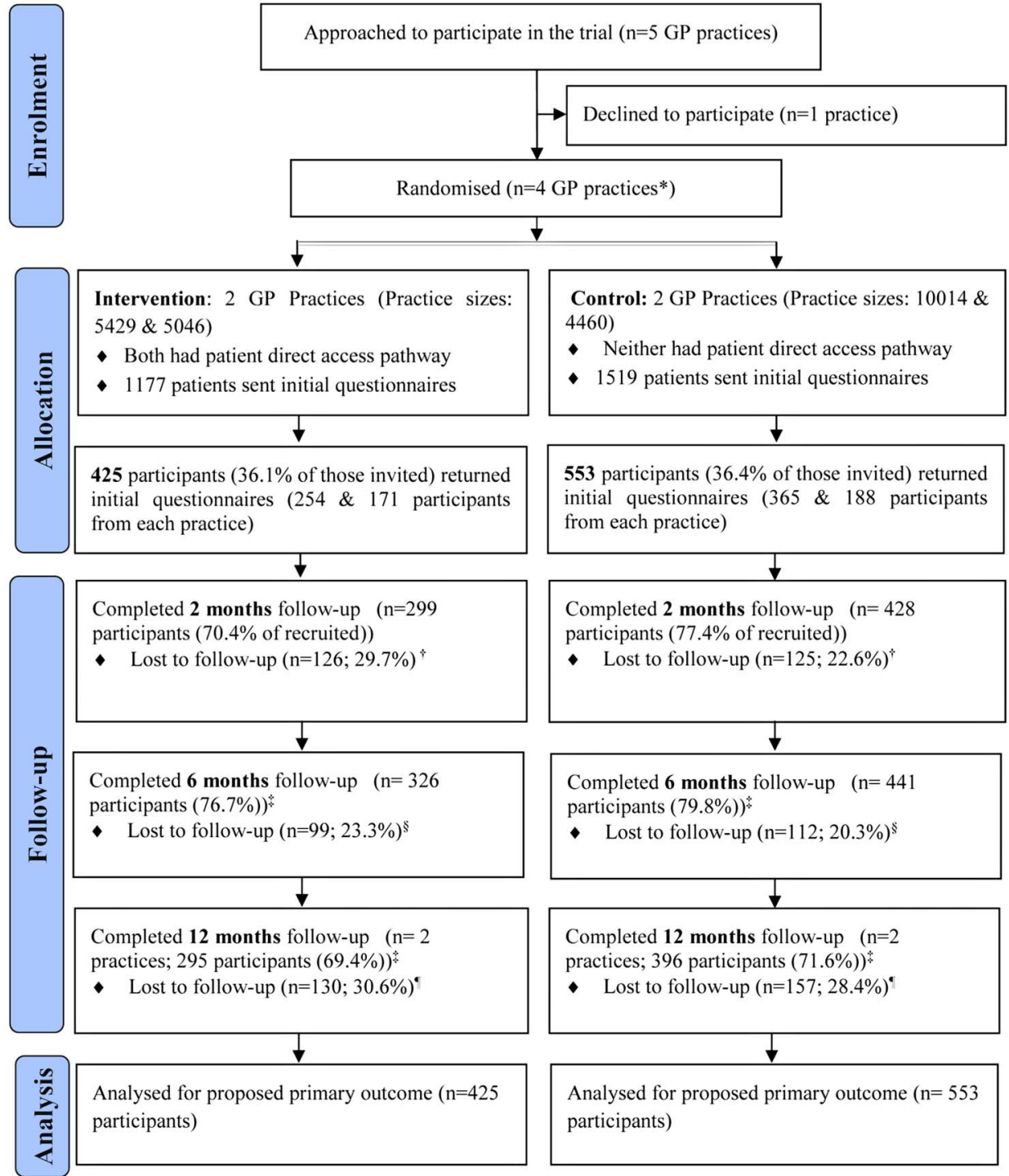

*All four randomised GP practices remained in the trial to the end

${ }^{*}$ Included 4 withdrawals, 2 from each arm

* The 6 and 12 months follow-up figures also include minimum data collection on key outcomes

$\$$ Included 42 additional withdrawals, 21 from each arm

"Included 15 additional withdrawals, 6 from intervention and 9 from control arm

Reasons for withdrawal were comparable between arms and included subsequently ineligible (2), not wishing to continue (24), questionnaire burden (3), ill health (8), death (5), bereavement (2), moving house (7), not having any MSK problem (4), time commitment (3), unable to communicate in English (1), wrong person completing questionnaire (1), and moving to care home (1).

\section{Comparability of baseline characteristics}

The baseline characteristics of participants (table 2) were comparable in the two arms of the trial and there was no evidence of selection bias.

\section{Baseline characteristics by physiotherapy access method}

The baseline characteristics of musculoskeletal consulters and study participants who did and did not access physiotherapy care in each trial arm are shown in online supplementary table S1. This shows that the characteristics of patients referred to physiotherapy by their GP or who accessed physiotherapy through self-referral were similar. However, 'true' self-referrers were more likely to be female and less likely to be in paid work. In addition, those in the most socioeconomically deprived group were less likely to use 'true' self-referral, although the numbers were very small.

\section{Research objectives}

Exploratory analysis of key clinical outcomes

The descriptive statistics (mean and SD or frequency and \%) for outcomes at each data collection time point are displayed in table 3 .

Patients' physical health measured using the SF-36 PCS is the anticipated primary clinical outcome for a future main trial. Higher scores represent better physical 
Table 2 Comparability of baseline characteristics

\begin{tabular}{|c|c|c|c|}
\hline Key characteristics & $\mathbf{n}^{*}$ & $\begin{array}{l}\text { Control } \\
\mathrm{n}=553\end{array}$ & $\begin{array}{l}\text { Intervention } \\
\mathrm{n}=425\end{array}$ \\
\hline \multicolumn{4}{|l|}{ Non-clinical characteristics } \\
\hline Age, mean (SD) & 978 & $58.6(14.6)$ & $55.6(15.2)$ \\
\hline Gender: female, n (\%) & 978 & $311(56.2)$ & $248(58.4)$ \\
\hline Ethnicity: white, $\mathrm{n}(\%)$ & 958 & 534 (99.3) & $416(99.1)$ \\
\hline Employment: in paid work (part-time/full-time)†, n (\%) & 966 & $244(44.4)$ & $190(45.2)$ \\
\hline $\begin{array}{l}\text { Extent to which pain has affected performance at work in the past } 1 \text { month } \\
\text { (0-10 NRS), mean (SD) }\end{array}$ & 434 & $4.8(3.2)$ & $4.9(3.1)$ \\
\hline Time off work (past month) due to pain, $\mathrm{n}(\%)$ & 407 & $74(32.3)$ & $54(30.3)$ \\
\hline Education: no qualifications, $\mathrm{n}(\%)$ & 849 & $133(27.7)$ & $104(28.2)$ \\
\hline Deprivation tertile (Index of Multiple Deprivation), n (\%) & 978 & & \\
\hline Most deprived third & & $161(29.1)$ & $103(24.2)$ \\
\hline Middle third & & $184(33.3$ & $145(34.1)$ \\
\hline Least deprived third & & $208(37.6)$ & $177(41.7)$ \\
\hline Health literacy: finds health information/leaflets difficult to understand, $n(\%)$ & 971 & $311(56.7)$ & $242(57.4)$ \\
\hline \multicolumn{4}{|l|}{ Clinical characteristics } \\
\hline Physical health: SF-36 PCS, mean (SD) & 952 & $35.7(10.1)$ & $36.6(9.9)$ \\
\hline Mental health: SF-36 MCS, mean (SD) & 952 & $44.4(13.7)$ & $42.7(14.0)$ \\
\hline Bodily pain past week: severe/very severe, n (\%) & 976 & $245(44.5)$ & $190(44.7)$ \\
\hline Current episode duration: $>6$ weeks, $n(\%)$ & 938 & $386(73.0)$ & $312(76.3)$ \\
\hline Widespread pain, $\ddagger$ n (\%) & 978 & $193(34.9)$ & $137(32.2)$ \\
\hline Comorbidities§: for $\geq 2$ other conditions, $n$ (\%) & 978 & $333(60.2)$ & $241(56.7)$ \\
\hline Quality of life: EQ-5D-5L, mean (SD) & 933 & $0.530(0.27)$ & $0.521(0.28)$ \\
\hline Pain self-efficacy (0-60), mean (SD) & 895 & $33.4(16.5)$ & $32.5(16.3)$ \\
\hline \multicolumn{4}{|c|}{$\begin{array}{l}\text { *Nine hundred and seventy-eight participants completed the baseline questionnaire but the number of respondents for each question slightly } \\
\text { varies due to missing data and non-applicable cases. } \\
\text { †Majority of those not in paid work (34\%) were retired from paid work. } \\
\text { fBased on the American College of Rheumatology's definition. }{ }^{21} \\
\S \text { The conditions include high blood pressure, heart problems, stroke, diabetes, depression or anxiety, osteoporosis, arthritis, asthma or } \\
\text { bronchitis, liver disease, cancer and others. } \\
\text { EQ-5D-5L, five-level version of the EuroQoL-5D; NRS, Numerical Rating Scale; SF- } 36 \text { MCS, } 36 \text {-item Short Form Mental Component } \\
\text { Summary-the higher the score the greater mental health; SF-36 PCS, } 36 \text {-item Short Form Physical Component Summary-the higher the } \\
\text { score the greater physical health. Pain self-efficacy scale: } 10 \text {-item scale, score range=0-60 (0=not at all confident, } 60=\text { completely confident } \\
\text { despite pain). }\end{array}$} \\
\hline
\end{tabular}

health. In this exploratory analysis very similar scores over time in participants in both arms of the trial were seen. The adjusted mean difference between groups and 95\% CI showed estimates of effect at 2 months -0.14 (95\% CI -1.02 to 0.74$)$, at 6 months -0.28 ( -1.35 to $0.79)$ and at 12 months $0.12(-1.27$ to 1.51$)$. The lower limit of the two-sided $95 \%$ CI for intervention versus control mean difference was $>-2$, the smallest expected standard treatment effect for the outcome (SF-36 PCS). Exploratory adjusted estimates of treatment effects for other outcomes are provided in the online supplementary table S2.

\section{Health economics results}

EQ-5D-5L responses were available at all time points for 493 participants $(281(51 \%)$ control arm, $212(50 \%)$ intervention arm). Self-reported resource use data were available at all time points for 555 participants (237 (43\%) control arm, 318 (57\%) intervention arm). Details of musculoskeletal-related NHS and healthcare resource use are reported in online supplementary table S3, with most resource use involving consultations with GPs, physiotherapists and practice nurses. Over 12 months, on average, the control arm was associated with slightly more GP visits, more investigations (including scans, X-rays and MRIs) and visits to some consultants (rheumatologists and orthopaedic surgeons) and inpatient days resulting from surgeries. The intervention arm was however associated with more physiotherapist visits.

Table 4 shows the disaggregated mean (SD) healthcare costs per patient for each treatment arm. The mean NHS costs per patient were slightly cheaper in the intervention arm ( $£ 940$ in the intervention compared with $£ 951$ in the control arm), with considerable uncertainty in the estimates $(95 \%$ CI of difference -363.27 to 340.83). The proportion of participants who reported having taken time off work due to their musculoskeletal problem was similar between the two arms of the trial over 12 months. The intervention arm was however associated with fewer self-reported days off work and lower costs at 12 months follow-up. Productivity loss and costs as a result of reduced productivity at work were higher in the intervention group. Mean quality of life scores (EQ-5D-5L; quality of life at baseline and follow-up and mean QALYs) are presented in table 5. Quality of life increased in both groups from the initial questionnaire 


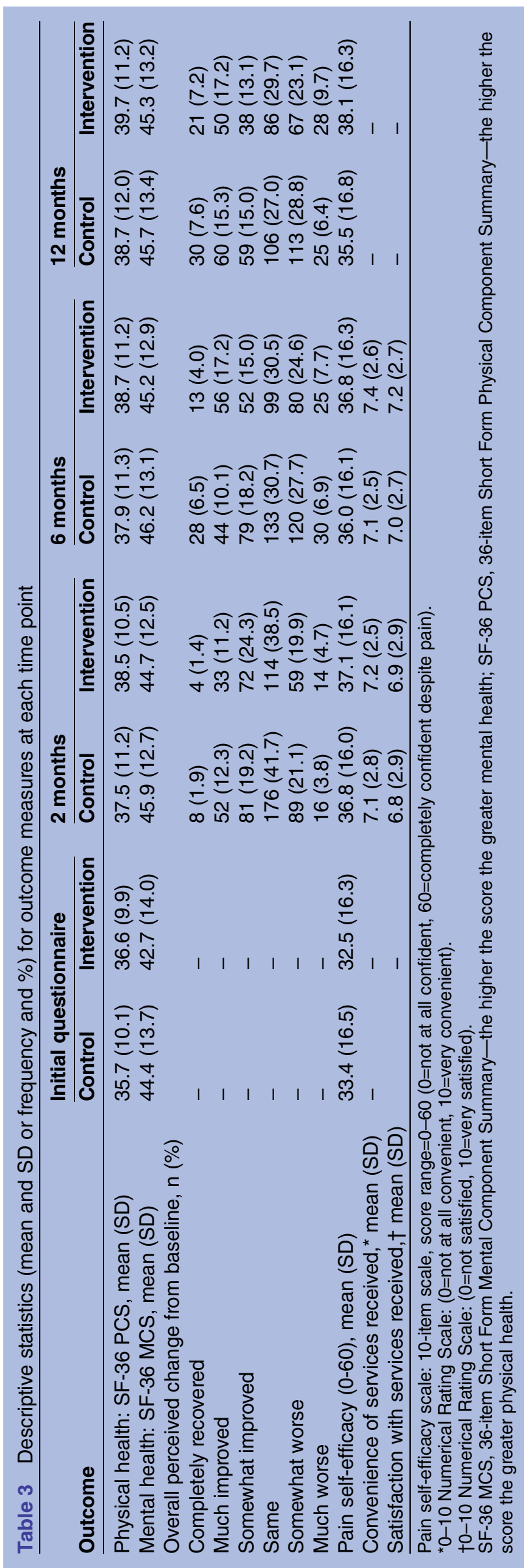

over all follow-up time points. Unadjusted mean QALYs were slightly higher in the control arm however, QALYs were similar in both groups arm once adjusted for clustering and baseline EQ-5D-5L score. Overall, the results show similar outcomes and costs between the intervention and control arms of the trial.

\section{DISCUSSION}

No other RCTs of direct access to physiotherapy have been conducted. This pilot RCT explored the feasibility of a future main cluster RCT and has provided an exploratory analysis of clinical and cost outcomes.

The results show that a future main trial is feasible and general practices and physiotherapy services are willing to take part. The patient recruitment target was achieved rapidly within the estimated 6-month recruitment period. Follow-up rates were good although the follow-up at 12 months fell below our anticipated $80 \%$. In a future main trial we would include small patient payments to compensate participants for their time in completing and returning questionnaires. Cluster trials have particular challenges including the concern about selection bias. ${ }^{22}{ }^{23}$ To minimise the risk of selection bias, we avoided participating clinicians being involved in the identification and recruitment of potential participants. The baseline characteristics of the participants showed no evidence of selection bias, which shows that our methods of identification and recruitment are suitable for use in a future main trial. We also compared the characteristics (age, gender and area-level deprivation) of those who returned the questionnaires and consented to data collection to those who did not return the questionnaires. These were comparable in terms of gender ( $57 \%$ vs $55 \%)$, but slightly different in terms of age (mean (SD) of 57 (15) vs 49 (17)) and deprivation (27\% most deprived third vs $38 \%$ for those who returned the questionnaires compared with those who did not). Participant recruitment and retention through long-term follow-up at 12 months was feasible and completed on time and to target. The likely primary clinical outcome for a main trial is patients' physical health assessed using the SF-36 PCS. ${ }^{13}$ This performed well in the pilot trial and exploratory analyses of clinical outcomes showed very similar changes in patient selfreported physical health measured in both arms of the trial. The hypotheses for a main trial would be noninferiority on clinical outcomes (ie, the addition of a patient direct access pathway to usual GP-led care would not lead to worse clinical outcomes for patients than usual GP-led care alone) but superiority on other key outcomes (ie, higher patient ratings of access to services, convenience and satisfaction with services, and reduced costs). A future main cluster RCT with a primary clinical outcome of physical health measured using the SF-36 PCS would require a sample size of $\sim 12$ average-sized general practices and a minimum of 2880 individual participants. This is based on a margin of non-inferiority of 
Table 4 Costs per participant by trial arm over 12 months

\begin{tabular}{|c|c|c|}
\hline Complete case analysis & $\begin{array}{l}\text { Control }(n=318) \\
\text { Emean (SD) }\end{array}$ & $\begin{array}{l}\text { Intervention }(\mathrm{n}=237) \\
\text { Emean (SD) }\end{array}$ \\
\hline \multicolumn{3}{|l|}{$\mathrm{NHS}^{*}$} \\
\hline GP & $106.70(134.57)$ & $96.97(134.58)$ \\
\hline Practice nurse & 7.87 (33.51) & 8.35 (37.93) \\
\hline Physiotherapist & $61.99(165.96)$ & $74.67(163.36)$ \\
\hline Orthopaedic surgeon & $48.43(114.47)$ & $36.58(102.54)$ \\
\hline Rheumatologist & $20.91(87.79)$ & $11.22(52.17)$ \\
\hline Acupuncturist & $5.67(50.74)$ & $1.67(13.63)$ \\
\hline Chiropractor & $4.28(35.81)$ & $1.48(15.08)$ \\
\hline Occupational therapist & $7.89(51.29)$ & $5.01(24.62)$ \\
\hline Other healthcare professionals & $33.88(187.28)$ & $21.67(117.81)$ \\
\hline Prescriptions & $55.18(118.24)$ & $47.15(110.41)$ \\
\hline Surgery & $422.97(1759.25)$ & $404.12(1655.21)$ \\
\hline Inpatient costs & $21.57(230.41)$ & $75.11(956.32)$ \\
\hline Investigations & 109.53 (329.93) & $100.77(263.43)$ \\
\hline Injections & 44.37 (164.06) & $55.22(274.33)$ \\
\hline \multicolumn{3}{|l|}{ Private* } \\
\hline Physiotherapist & $9.46(55.05)$ & $22.68(155.41)$ \\
\hline Orthopaedic surgeon & 4.49 (34.07) & $3.01(23.74)$ \\
\hline Rheumatologist & 0 & $1.12(17.27)$ \\
\hline Acupuncturist & $3.04(30.91)$ & $5.76(40.60)$ \\
\hline Chiropractor & $9.13(85.47)$ & $6.68(48.31)$ \\
\hline Occupational therapist & 0 & $0.74(11.43)$ \\
\hline Other healthcare professionals & $3.98(38.49)$ & $7.63(97.90)$ \\
\hline Over-the-counter medicines & $20.20(52.59)$ & $15.06(30.06)$ \\
\hline \multicolumn{3}{|l|}{ Work-related outcomes } \\
\hline Musculoskeletal-related work absence costs & $740.30(2084.75)$ & $539.36(2069.43)$ \\
\hline \multicolumn{3}{|l|}{ Complete case } \\
\hline Total NHS cost, $£$ & $951.25(2050.88)$ & $940.02(2157.24)$ \\
\hline Mean difference $(95 \% \mathrm{Cl}) \dagger$ & $11.22(-363.27$ to 340.82$)$ & \\
\hline Total healthcare costs, $\ddagger £$ & $1001.56(2068.62)$ & $1002.72(2230.66)$ \\
\hline Mean difference $(95 \% \mathrm{Cl}) \dagger$ & $-1.15(-358.00$ to 360.32$)$ & \\
\hline
\end{tabular}

Table 5 EQ-5D-5L scores and QALYs at each time point

\begin{tabular}{lcc} 
Complete case analysis & $\begin{array}{l}\text { Control (n=281) } \\
\text { mean (SD) }\end{array}$ & $\begin{array}{l}\text { Intervention } \\
\text { (n=212) mean (SD) }\end{array}$ \\
\hline Initial questionnaire & $0.565(0.246)$ & $0.544(0.262)$ \\
2 months & $0.600(0.227)$ & $0.580(0.246)$ \\
6 months & $0.602(0.251)$ & $0.594(0.262)$ \\
12 months & $0.615(0.254)$ & $0.606(0.258)$ \\
Total 12 months QALYs (unadjusted) & $0.602(0.225)$ & $0.589(0.236)$ \\
Incremental QALYs (95\% Cl)* & $-0.012(-0.053$ to 0.028$)$ & 0.578 \\
Total 12 months QALYs (adjusted for clustering and baseline EQ-5D-5L)† & 0.575 & 0.003 \\
Incremental QALYs & & \\
\hline${ }^{*}$ Difference=intervention-control. Cls were generated using regression models for clustered data. & \\
†QALYs have been adjusted for baseline differences and clustering. & \\
EQ-5D-5L, five-level version of the EuroQol-5D; QALYs, quality-adjusted life years. & &
\end{tabular}

2 points from previous research, ${ }^{17-20}$ a SD of 10 , a power of $90 \%$ at a $2.5 \%$ one-sided significance level, in a design with three repeated measurements ${ }^{24}$ and an autocorrelation of 0.5 . The sample size has also been inflated by $20 \%$ to allow for loss to follow-up, and a further inflation for the design effect using an ICC of $0.0075,{ }^{25}$ and an average cluster size of 240 , allowing for a coefficient of variation in cluster size of $0.65 .^{26}$

Exploratory health economic findings show that costs and outcomes were similar between the intervention 
and control arms, although there is considerable imprecision in the estimates due to the sample size in this pilot RCT. Data collection through patient questionnaires was successful and indicates that a full economic evaluation of adding a patient direct access to physiotherapy pathway to usual GP-led primary care versus usual GP-led primary care alone will be possible in a future main trial. Additional costs required to set-up the service should also be considered in a future trial.

This pilot trial suggests that adding a new patient direct access pathway to an existing physiotherapy service is feasible. We observed an increase in the number of overall referrals to physiotherapy in the intervention arm practices compared with service-level data collected in the year prior to the STEMS pilot trial, mostly from one practice, but this did not impact on the waiting time for physiotherapy care. The level of GP referral to physiotherapy prior to the STEMS pilot trial was very low in this one practice compared with the other practices. Pre-existing referral rates to physiotherapy have previously been highlighted as a possible contributing factor to subsequent increases in demand following the introduction of patient direct access. ${ }^{27}$ An initial increase in referrals is perhaps not surprising bearing in mind the active marketing of the direct access pathway that was undertaken. This type of marketing is unlikely to occur outside the context of a trial but in a future main trial processes should be in place to support participating physiotherapy services through availability of suitably trained staff and resources for increasing staffing levels if demand increases. While no robust claims can be made about safety given the pilot nature of this RCT, no adverse events were reported. In addition, our systematic search of diagnostic codes of serious or significant pathology in the medical records of patients who directly accessed physiotherapy found no evidence that the direct access pathway led to missed serious pathologies. This is in keeping with a recent analysis of 12976 patient visits to a US physical therapist-led student health centre over 10 years, without a physician referral that reported no adverse events and no missed cases of serious pathology. ${ }^{28}$

\section{Non-attendance rates}

We also aimed to compare patient non-attendance rates for physiotherapy before and during patient direct access, but we were unable to achieve this as the physiotherapy service changed the definition and processes for recording non-attendance part way through the STEMS pilot trial.

\section{CONCLUSION}

In line with calls for research on ways to ensure patient demand can be met in primary care, this pilot trial shows that a future large RCT is feasible and will provide high-quality evidence about the clinical and costeffectiveness of direct access to physiotherapy for patients with musculoskeletal pain.
Author affiliations

${ }^{1}$ Research Institute for Primary Care \& Health Sciences, Keele University, Keele, UK

${ }^{2}$ Health Economics Unit, University of Birmingham, Birmingham, UK ${ }^{3}$ Centre for Academic Primary Care, University of Bristol, Bristol, UK

Acknowledgements The STEMS trial team acknowledges the support from Keele Clinical Trials Unit, including Dr Ruth Beardmore (Research Programme Manager) for input into research governance and gaining of regulatory approvals, and administrators Michelle Robinson and Victoria Harper for coordinating mailings of consenting patients and general research administrative support. The authors also acknowledge the NIHR Clinical Research Network (CRN) including lan Thomas for coordinating systems to help the participating practices identify eligible patients and assistance in providing anonymised patient data, the research nurse team, including Julie Young, Debbie D'Cruz, Alice Mackie, William Cole and Sian Jones who dealt with patient queries and conducted minimum data collection via telephone. The authors thank the CRN physiotherapy and GP research facilitators, Gemma Sellors, Carol Doyle and Beth Hanson. The authors also thank the Independent Advisory Panel comprising Jill Gamlin, Sue Greenhalgh, Lesley Holdsworth and Ruth ten Hove who provided advice on setting up patient direct access to physiotherapy and Sue Greenhalgh for her input into the physiotherapist training. The authors are grateful to the general practices and physiotherapy service that participated in this pilot trial and in particular the support of Ruth Heaton, Therapy Manager, and to the physiotherapists who delivered care to patients. The authors acknowledge Arthritis Research UK who funded early stakeholder thinktanks to develop and design the STEMS trial. Finally, the authors are particularly indebted to the PPIE members, Trial Steering Committee members and all the participants who provided data.

Contributors $A B$ helped plan the trial, contributed to the funding application, chaired the Trial Management Group (TMG) and led the writing of this paper. ROO developed the analysis plan and performed the quantitative analyses. SJ developed the health economic data collection and analysis plan and supervised JK who conducted the health economic analysis. ST was responsible for trial coordination, supporting the TMG, the general practices and physiotherapy service. JP provided general practice expertise and contributed to the training of physiotherapists offering the direct access pathway. EMH provided senior trials leadership, expertise and oversight. CS helped design the trial and provided general practice expertise. NEF led the design of the trial and the funding application, and provided senior trials leadership and oversight. All authors contributed to this paper and approved the final version.

Funding The STEMS pilot trial was funded by the Chartered Society of Physiotherapy Charitable Trust (SRP (11)2). NEF and AB are funded through a National Institute of Health Research (NIHR) Research Professorship awarded to NEF (NIHR-RP-011-015). NEF is an NIHR senior investigator. The North Staffordshire Primary Care Research Consortium provided additional funding for GP and Physiotherapy Research Facilitators to advise on the engagement and recruitment of GP practices and physiotherapists in the STEMS pilot trial.

Disclaimer The views and opinions expressed are those of the authors and do not necessarily reflect those of the NHS, the NIHR or the Department of Health.

Competing interests None declared.

Ethics approval NRES North West-Preston Preston in February 2013 (REC reference 13/NW/0053).

Provenance and peer review Not commissioned; externally peer reviewed.

Data sharing statement No additional data are available.

Open Access This is an Open Access article distributed in accordance with the Creative Commons Attribution Non Commercial (CC BY-NC 4.0) license, which permits others to distribute, remix, adapt, build upon this work noncommercially, and license their derivative works on different terms, provided the original work is properly cited and the use is non-commercial. See: http:// creativecommons.org/licenses/by-nc/4.0/ 


\section{REFERENCES}

1. Roland $\mathrm{M}$, Everington $\mathrm{S}$. Tackling the crisis in general practice. $B M J$ 2016;352:i942.

2. Hobbs FDR, Bankhead C, Mukhtar T, et al. on behalf of the National Institute for Health Research School for Primary Care Research. Clinical workload in UK primary care: a retrospective analysis of 100 million consultations in England, 2007-14. Lancet 2016;387:2323-30.

3. Jordan KP, Kadam UT, Hayward R, et al. Annual consultation prevalence of regional musculoskeletal problems in primary care: an observational study. BMC Musculoskelet Disord 2010;11:144.

4. Murray CJ, Vos T, Lozano R, et al. Disability-adjusted life years (DALYs)for 291 diseases and injuries in 21 regions, 1990-2010: a systematic analysis for the global burden of disease study 2010. Lancet 2012;380:2197-223.

5. Holdsworth LK, Webster VS, McFadyen AK. What are the costs to NHS Scotland of self-referral to physiotherapy? Results of a national trial. Physiotherapy 2007:93:3-11.

6. Department of Health. Self-referral pilots to musculoskeletal physiotherapy and the implications of improving access to other AHP services. Leeds. Department of Health, 2008 http://www.dh.gov.uk/ en/Publicationsandstatistics/Publications/PublicationsPolicy AndGuidance/DH_089516. (accessed $01 \mathrm{Feb} 2015$ ).

7. Leemrijse CJ, Swinkels ICS, Veenhof C. Direct access to physical therapy in the Netherlands: results from the first year in community-based physical therapy. Phys Ther 2008;88:936-46.

8. QIPP Quality and Productivity Case Studies. Musculoskeletal physiotherapy: patient self-referral 2012 citing Holdsworth et al. 2007, costs updated in accordance with Department of Health 2011. https://www.evidence.nhs.uk/qipp (accessed 10 Jan 2015).

9. Mallett $R$, Bakker $E$, Burton $M$. Is physiotherapy self-referral with telephone triage viable, cost-effective and beneficial to musculoskeletal outpatients in a primary care setting? Musculoskeletal Care 2014;12:251-60.

10. The Kings Fund. The UK Private Health Market. 2014. https://www. kingsfund.org.uk/sites/files/kf/media/commission-appendix-ukprivate-health-market.pdf

11. Primary Care Workforce Commission. The future of primary care Creating teams for tomorrow. 2015. http://www.hee.nhs.uk

12. Bishop A, Tooth S, Protheroe J, et al. on behalf of the STEMS study team. A pilot cluster randomised controlled trial to investigate the addition of direct access to physiotherapy to usual GP-led primary care for adults with musculoskeletal pain: the STEMS pilot tria protocol (ISRCTN23378642). Pilot Feasibility Stud 2015;1:26.

13. SF-36.org: A community for measuring health outcomes using SF tools. http://www.sf-36.org/
14. Herdman M, Gudex C, Lloyd A, et al. Development and preliminary testing of the new five-level version of EQ-5D (EQ-5D-5L). Qual Life Res 2011;20:1727-36.

15. Nicholas MK. The pain self-efficacy questionnaire: taking pain into account. Eur J Pain 2007:11:153-63.

16. Mead N, Bower P, Roland M. The general practice assessment questionnaire (GPAQ): development and psychometric characteristics. BMC Fam Pract 2008;9:13.

17. Ware JE, Kosinski M, Bjorner JB, et al. User's manual for the SF-36v2 Health Survey. 2nd edn. Lincoln, RI:QualityMetric Inc, 2007.

18. Angst F, Aeschlimann A, Stucki G. Smallest detectable and minimal clinically important differences of rehabilitation intervention with their implications for required sample sizes using WOMAC and SF-36 quality of life measurement instruments in patients with osteoarthritis of the lower extremities. Arthritis Care Res 2001;45:384-91.

19. Kosinski M, Zhao SZ, Dedhiya S, et al. Determining minimally important changes in generic and disease-specific health-related quality of life questionnaires in clinical trials of rheumatoid arthritis. Arthritis Rheum 2000;43:1478-87.

20. Norman GR, Sloan JA, Wyrwich KW. Interpretation of changes in health-related quality of life: the remarkable universality of half a standard deviation. Med Care 2003;41:582-92.

21. Wolfe F, Smythe H, Yunus M, et al. The American College of Rheumatology 1990 criteria for the classification of fibromyalgia. Arthritis Rheum 1990;33:160-71.

22. Puffer S, Torgerson D, Watson J. Evidence for risk of bias in cluster randomised trials: review of recent trials published in three general medical journals. BMJ 2003;327:785-9.

23. Hahn S, Puffer S, Torgerson DJ, et al. Methodological bias in cluster randomised trials. BMC Med Res Methodol 2005;5:10.

24. Diggle PJ, Heagerty P, Liang K-Y, et al. Analysis of longitudinal data. 2nd edn. Oxford: Oxford University Press, 2002.

25. Adams G, Gulliford MC, Ukoumunne OC, et al. Patterns of intra-cluster correlation from primary care research to inform study design and analysis. J Clin Epidemiol 2004;57:785-94.

26. Eldridge SM, Ashby D, Kerry S. Sample size for cluster randomized trials: effect of coefficient of variation of cluster size and analysis method. Int J Epidemiol 2006;35:1292-300.

27. Salisbury C. http://ukpolicymatters.thelancet.com/making-the-mostof-evaluation-to-ensure-health-policy-reform-is-evidence-based/ 2011.

28. Mintken PE, Pascoe SC, Barsch AK, et al. Direct access to physical therapy services is safe in a university student health center setting. $J$ Allied Health 2015;44:164-8. 